\title{
LINEAR PROCESSES GENERATED BY INDEPENDENT RANDOM VARIABLES
}

\author{
W. A. WOYCZYŃSKI ${ }^{1}$
}

\begin{abstract}
In a recent paper $R$. Dudley gave a characterization of those sequences of independent and identically distributed random variables which are $l_{p}$-compatible for $p \geqq 1$. In the present note we extend his result into $p \in(0,1]$ and provide some conditions (necessary or sufficient) for $l_{\varphi}$-compatibility of a sequence of independent random variables not necessarily identically distributed.
\end{abstract}

Let $\left\{X_{n}\right\} \subset \mathscr{M}(\Omega, \mathscr{F}, P)$ be a sequence of random variables. If for every element $\left(a_{n}\right)$ of a complete metric linear space $\mathscr{X}$ of real sequences in which coordinate functionals $\left(a_{n}\right) \rightarrow a_{m}, m=1,2, \cdots$, are continuous, the series $\sum a_{n} X_{n}$ converges in probability (we shall say in this case that $\left\{X_{n}\right\}$ is $\mathscr{X}$ compatible), then the mapping

$$
X: \mathscr{X} \ni\left(a_{n}\right) \rightarrow \sum a_{n} X_{n} \in \mathscr{M}(\Omega, \mathscr{F}, P)
$$

defines a linear continuous stochastic process on $\mathscr{X}$ (for basic definitions the reader is referred to [1], [4] and references given therein). The linearity is clear and the continuity follows from the classical Banach theorem which says that every Borel homomorphism of a complete metric group into a metric is continuous and from a remark that $X$ is a pointwise limit of continuous (because finite-dimensional) hence Borel functions $\sum_{h=1}^{n_{0}} a_{n} X_{n}$. R. Dudley [2] gave a characterization of those sequences of independent and identically distributed random variables which are $l_{p}$-compatible for $p \geqq 1$. In this note we extend his result onto $p \in(0,1]$ and provide a necessary condition for the sequence of independent random variables (not necessarily identically distributed) to be $l_{\varphi}$-compatible and the sufficient condition for the same sequence to be $l_{p}$-compatible for $p>0$. However it seems that they are rather far from the conditions which are necessary and sufficient simultaneously.

Received by the editors August 2, 1971.

AMS 1970 subject classifications. Primary 60B05, 60B99.

Key words and phrases. Linear stochastic process, $l_{\varphi}$-compatible, weakly $\varphi$-finite.

${ }^{1}$ The author acknowledges support received under NSF Grant GU-2056, administered by Carnegie-Mellon University, while on leave from Wroclaw University and the Mathematical Institute of the Polish Academy of Sciences.

(C) American Mathematical Society 1972 
Now let $\left\{X_{n}\right\}$ be a sequence of independent random variables with distribution functions $F_{n}$ and let $\varphi, \psi: \boldsymbol{R}^{+} \rightarrow \boldsymbol{R}^{+}$be nondecreasing continuous functions vanishing only at the origin. Call the sequence $\left\{X_{n}\right\}$ (or $\left\{F_{n}\right\}$ ) lower [upper] weakly $\varphi$-finite if, for some $C>0$,

$$
\lim \inf _{n}\left[\sup _{n}\right] P\left\{\left|X_{n}\right| \geqq M\right\} \leqq C / \varphi(M), \quad 0 \leqq M<\infty .
$$

We shall say that $\varphi$ satisfies the $\Delta_{2}$-condition $\left(\varphi \in \Delta_{2}\right)$ if for some positive constant $K, \varphi(2 t) \leqq K \varphi(t), 0 \leqq t<\infty$. If $\varphi \in \Delta_{2}$ then the sequence space

$$
l_{\varphi} \equiv\left\{\left(a_{n}\right) \in \boldsymbol{R}^{\infty}: \sum \varphi\left(\left|a_{n}\right|\right)<\infty\right\}
$$

is a linear space which can be equipped with a nonhomogeneous norm (e.g. $\left.\left\|\left(a_{n}\right)\right\|_{\varphi}=\inf \left\{c: c>0, \sum_{\varphi}\left(\left|a_{n}\right| c \mid\right) \leqq c\right\}\right)$ which makes $l_{\varphi}$ a complete metric linear space with continuous coordinate functionals (see e.g. [3, Theorems 1.4, 1.62 and 1.82]).

The propositions given below explain the relationship between lower [upper] finiteness and the existence of moments for a sequence $\left\{X_{n}\right\}$.

Proposition 1. If, for some subsequence $\left\{n_{k}\right\}, k=1,2, \cdots, E \varphi\left(\left|X_{n_{k}}\right|\right)$ are finite and $\lim \inf _{k} E \varphi\left(\left|X_{n_{k}}\right|\right)=L<\infty$, then the sequence $\left\{X_{n}\right\}$ is lower weakly $\varphi$-finite.

Proof.

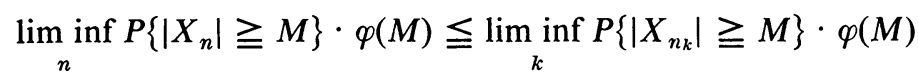

$$
\leqq \liminf _{k} \int_{\left|X_{n_{k} \mid}\right| \geqq M} \varphi\left(\left|X_{n_{k}}\right|\right) d P \leqq \lim _{k} \inf E \varphi\left(\left|X_{n_{k}}\right|\right)=L<\infty .
$$

Proposition 2. Let $\varphi \in \Delta_{2}, \varphi, \psi$ be differentiable with monotone $\varphi^{\prime}$, and let $\psi(t) \varphi^{-1}(t) \rightarrow 0$ as $t \rightarrow+\infty$ in such a way that $\psi(t) \varphi^{-1}(t) t^{-1} \in L^{1}(\alpha, \infty)$ for some $\alpha>0$. In those conditions if $\varphi(M) \lim \sup _{n} P\left\{\left|X_{n}\right| \geqq M\right\} \leqq C, 0 \leqq M<$ $\infty$ (even more so if $\left\{X_{n}\right\}$ is upper weakly $\varphi$-finite) then $\lim \sup _{n} E \psi\left(\left|X_{n}\right|\right)<\infty$.

Proof. Note that

$$
\begin{aligned}
& \underset{n}{\lim \sup } E \psi\left(\left|X_{n}\right|\right)=\lim \sup _{n} \int_{-\infty}^{\alpha} \psi(|t|) d F_{n}(t) \\
& +\lim \sup \int_{\left|X_{n}\right| \leqq \alpha}^{n} \psi\left(\left|X_{n}\right|\right) d P+\lim \sup _{n} \int_{\alpha}^{\infty} \psi(|t|) d\left(F_{n}(t)-1\right) .
\end{aligned}
$$

The second term being bounded by $\psi(\alpha)$ it is sufficient to evaluate the first and the third ones. We restrict ourselves to the evaluation of the third term (the procedure for the first one is analogous). Integrating by parts we 
get that

$$
\begin{aligned}
\lim \sup _{n} \int_{\alpha}^{\infty} \psi(t) & d\left(F_{n}(t)-1\right) \\
= & \limsup _{n}\left[\frac{\psi(t)}{\varphi(t)} \varphi(t)\left(F_{n}(t)-1\right)\right]_{\alpha}^{\infty}+\int_{\alpha}^{\infty} \psi^{\prime}(t)\left(1-F_{n}(t)\right) d t .
\end{aligned}
$$

By our assumptions, $\psi(t) \cdot \varphi^{-1}(t) \rightarrow 0$ as $t \rightarrow \infty$ and $\lim _{\sup _{n}} \varphi(t)\left(F_{n}(t)-1\right)$ remains bounded. Hence the first summand is bounded by $\psi(\alpha)$. As to the second one we have that

$$
\lim \sup _{n} \int_{\alpha}^{\infty} \psi^{\prime}(t)\left(1-F_{n}(t)\right) d t \leqq C \int_{\alpha}^{\infty} \frac{\psi^{\prime}(t)}{\varphi(t)} d t \leqq C \int_{\alpha}^{\infty} \frac{\psi(t)}{\varphi(t) t} \frac{\varphi^{\prime}(t) t}{\varphi(t)} d t
$$

We shall prove that $\varphi^{\prime}(t) t / \varphi(t)$ is bounded so that the last integral is finite. Indeed, when $\varphi^{\prime}$ is increasing then (because of the $\Delta_{2}$-condition)

$$
K \varphi(t) \geqq \varphi(2 t)=\int_{0}^{2 t} \varphi^{\prime}(\tau) d \tau>\int_{t}^{2 t} \varphi^{\prime}(\tau) d \tau>t \varphi^{\prime}(t)
$$

and in the case $\varphi^{\prime}$ is decreasing

$$
\varphi(t)=\int_{0}^{t} \varphi^{\prime}(\tau) d \tau>\int_{t / 2}^{t} \varphi^{\prime}(\tau) d \tau>\frac{1}{2} t \varphi^{\prime}(t) . \quad \text { Q.E.D. }
$$

Remarks. From the Proposition 2 and its proof it follows that

(a) if $\varphi, \psi \in \Delta_{2}$ have both monotone derivatives, $\psi(t) \varphi^{-1}(t) \rightarrow 0$ as $t \rightarrow+\infty$ in such a way that $\psi^{\prime}(t) / \varphi(t) \in L^{1}(\alpha, \infty)$ for some $\alpha>0$, and $\left\{X_{n}\right\}$ is upper weakly $\varphi$-finite then $\lim \sup _{n} E \psi\left(\left|X_{n}\right|\right)<\infty$.

(b) If $\left\{X_{n}\right\}$ is upper weakly $\varphi$-finite with $\varphi(u)=u^{p}, p>0$, then

for every $q, 0<q<p$.

$$
\lim _{n} \sup E\left|X_{n}\right|^{q}<\infty
$$

In what follows we shall need the following condition for the function $\varphi$ :

$$
0<\text { const } \leqq \varphi(t) \varphi\left(t^{-1}\right) \leqq \operatorname{CONST}<\infty .
$$

As examples of such a function we may give $\varphi(t)=t^{p}, p>0$, and

$$
\begin{array}{rlrl}
\varphi(t) & =\int_{0}^{t} \frac{|\sin u|}{u} d u & \text { for } 0<t \leqq \pi, \\
& =\varphi(\pi)+\int_{\pi}^{t}|\sin u| d u & & \text { for } \pi<t .
\end{array}
$$

THEOREM 1. Let $\varphi \in \Delta_{2}$ satisfy condition $(+)$. In this case if $\left\{X_{n}\right\}$ is $l_{\varphi}$ compatible then it is lower weakly $\varphi$-finite. If in addition $\varphi^{\prime}$ exists and is 
monotone then $l_{\varphi}$-compatibility of $\left\{X_{n}\right\}$ implies that $\varphi^{\prime}(t) \lim \inf _{n} \int_{-t}^{t} s d F_{n}(s)$ is bounded for $0 \leqq t<\infty$.

Proof. Assume that $\left\{F_{n}\right\}$ is not lower weakly $\varphi$-finite. Then for every $k=1,2, \cdots$, we can find $M_{k}$ such that

$$
\min \left(\varphi\left(M_{k}\right), M_{k}\right) \geqq k^{2} \quad \text { and } \quad \varphi\left(M_{k}\right) \lim \inf P\left(\left|X_{n}\right| \geqq M_{k}\right)>k \text {. }
$$

It follows that for every $k=1,2, \cdots$, there exists an integer $N_{k}$ such that

$$
P\left(\left|X_{j}\right| \geqq M_{k}\right)>k / \varphi\left(M_{k}\right), \quad j \geqq N_{k} .
$$

Let now $a_{j}=1 / M_{k}$ for $m_{k} \leqq j<m_{k}+r_{k}$, where $m_{1}=N_{1}$,

$$
m_{k+1}=\max \left(m_{k}+r_{k}, N_{k+1}\right)
$$

and where $r_{k}$ is an integer such that

$$
1 \leqq k^{2} r_{k} / \varphi\left(M_{k}\right) \leqq 2,
$$

and $a_{j}=0$ for $j$ 's not listed above (if any). We shall check that $\left(a_{j}\right) \in l_{\varphi}$. Indeed

$$
\sum_{j} \varphi\left(\left|a_{j}\right|\right)=\sum_{k} r_{k} \varphi\left(\frac{1}{M_{k}}\right) \leqq \operatorname{CONST} \sum_{k} \frac{r_{k}}{\varphi\left(M_{k}\right)} \leqq \operatorname{CONST} \sum_{k} \frac{2}{k^{2}}<\infty
$$

because of the condition $(+)$. However $(*)$ and $(* *)$ cause the series

$$
\sum_{j} P\left(\left|a_{j} X_{j}\right| \geqq 1\right)=\sum_{k=1}^{\infty} \sum_{j=m_{k}}^{m_{k}+r_{k}-1} P\left(\left|X_{j}\right| \geqq M_{k}\right) \geqq \sum_{k=1}^{\infty} \frac{r_{k} k}{\varphi\left(M_{k}\right)} \geqq \sum_{k=1}^{\infty} \frac{1}{k}
$$

to be divergent, causing the divergence of $\sum_{j} a_{j} X_{j}$ in probability by the Kolmogorov three series theorem.

Now assume $\varphi^{\prime}$ is monotone and $\varphi^{\prime}(t) \lim \inf _{n} \int_{-t}^{t} s d F_{n}(s)$ is unbounded on the positive half-line. Then, as in the preceding part of the proof, for every $k=1,2, \cdots$, we can find $M_{k}, N_{k}$ such that $\min \left(\varphi\left(M_{k}\right), M_{k}\right) \geqq k^{2}$ and

$(* * *)$

$$
I_{j k}=\varphi^{\prime}\left(M_{k}\right) \int_{|t| \leqq M I_{k}} s d F_{j}(s)>k, \quad j \geqq N_{k}
$$

(or $-I_{j k}>k$; in this case we proceed similarly). Having chosen $\left(a_{j}\right)$ as previously in $l_{\varphi}$, we see in view of $(* * *)$ that $^{2}$

$$
\begin{aligned}
\sum_{j} E\left(a_{j} X_{j}\right)^{1} & =\sum_{k=1}^{\infty} \frac{1}{M_{k}} \sum_{j=m_{k}}^{m_{k}+r_{k}-1} \int_{-M_{k}}^{M_{k}} s d F_{j}(s)>\sum_{k=1}^{\infty} \frac{r_{k} k}{M_{k} \varphi^{\prime}\left(M_{k}\right)} \\
& \geqq \frac{1}{\max (2, K)} \sum_{k=1}^{\infty} \frac{r_{k} k}{\varphi\left(M_{k}\right)} \geqq \frac{1}{\max (2, K)} \sum_{k=1}^{\infty} \frac{1}{k},
\end{aligned}
$$

${ }^{2} X^{1}(\omega)$ is equal to $X(\omega)$ if $|X(\omega)| \leqq 1$ and 0 otherwise. 
the penultimate inequality being motivated as in the proof of the Proposition 2 by the $\Delta_{2}$-condition and the monotonicity of $\varphi^{\prime}$. Thus again by the Kolmogorov three series theorem we conclude that $\left\{X_{j}\right\}$ is not $I_{\varphi}$-compatible. Q.E.D.

Corollary. If $\sup _{t} \varphi^{\prime}(t)=\infty$ (e.g. if $\left.\varphi(t)=t^{p}, p>1\right)$ and $\left\{X_{n}\right\}$ is $l_{\varphi^{-}}$ compatible then $\lim \inf _{n} E X_{n}=0$.

THEOREM 2. If $\left\{F_{n}\right\}$ is upper weakly $\varphi$-finite, where $\varphi(t)=t^{p}, 0<p<2$, and $t^{p-1} \sup _{n}\left|\int_{-t}^{t} s d F_{n}(s)\right| \leqq C^{\prime}<\infty, 0 \leqq t<\infty$, then $X_{n}$ is $l_{\varphi}$-compatible.

Proof. We know that $M^{p} \sup _{n} P\left(\left|X_{n}\right|>M\right)<C$ and we shall check the convergence of all three series in Kolmogorov's theorem. $\left(a_{i}\right)$ always is supposed to belong to $l_{p}$.

$$
\begin{aligned}
\sum_{i} P\left(\left|a_{i} X_{i}\right|>1\right) & \leqq \sum_{i} P\left(\left|X_{i}\right|>\frac{1}{\left|a_{i}\right|}\right) \leqq \sum_{i} \sup _{n} P\left(\left|X_{n}\right|>\frac{1}{\left|a_{i}\right|}\right) \\
& \leqq C \sum_{i}\left|a_{i}\right|^{p}<\infty . \\
\sum_{i}\left|E\left(a_{i} X_{i}\right)^{1}\right| & =\sum_{i}\left|a_{i}\right|\left|\int_{|t|<1 /\left|a_{i}\right|} s d F_{i}(s)\right| \\
& \leqq \sum_{i} a_{i} \sup _{n}\left|\int_{|t|<1 /\left|a_{i}\right|} t d F_{n}\right| \\
& \leqq C^{\prime} \sum_{i}\left|a_{i}\right|\left|a_{i}\right|^{p-1}<\infty . \\
\sum_{i} E\left(\left(a_{i} X_{i}\right)^{1}\right)^{2} & =\sum_{i} a_{i}^{2} \int_{|t|<1 /\left|a_{i}\right|} t^{2} d F_{i}(d t) .
\end{aligned}
$$

It is sufficient to evaluate the series

$$
\begin{aligned}
\sum_{i} a_{i}^{2} \int_{0}^{1 /\left|a_{i}\right|} t^{2} d F_{i}(t) & \leqq \sum_{i} a_{i}^{2}\left(\frac{1}{a_{i}^{2}}-2 \int_{0}^{1 /\left|a_{i}\right|} t F_{i}(t) d t\right) \\
& =2 \sum_{i} a_{i}^{2} \int_{0}^{1 /\left|a_{i}\right|} t\left(1-F_{i}(t)\right) d t \\
& \leqq 2 \sum_{i} a_{i}^{2}\left(1+C \int_{1}^{1 /\left|a_{i}\right|} t^{1-p} d t\right) \\
& =2 \sum_{i}\left(1-\frac{C}{2-p}\right) a_{i}^{2}+\frac{2 c}{2-p} \sum_{i}\left|a_{i}\right|^{p}<\infty
\end{aligned}
$$

REMARKS. (1) If the random variables $\left\{X_{n}\right\}$ are identically distributed with distribution function $F$ then the Theorems 1 and 2 give the following corollary which is the extension of R. Dudley's Theorem 7.2 of 
[2]: if $0<p<2$ then $X_{n}$ is $l_{p}$-compatible if and only if $F$ is weakly $t^{p}$-finite (i.e. $M^{p} P\left(\left|X_{1}\right| \geqq M\right)$ is bounded) and $t^{p-1} \int_{-t}^{t} s d F(s)$ is bounded.

(2) For $p>1$ assumptions of Theorem 2 cause, clearly, $E X_{n}=0$.

\section{REFERENCES}

1. A. Badrikian, Séminaire sur les mesures cylindriques et les fonctions linéaires aléatoires, Lecture Notes in Math., no. 139, Springer-Verlag, Berlin and New York, 1970.

2. R. M. Dudley, Random linear functionals, Trans. Amer. Math. Soc. 136 (1969), 1-24. MR 41 \#9317.

3. S. Mazur, and W. Orlicz, On some classes of linear spaces, Studia Math. 17 (1958), 97-119. MR 20 \#4780.

4. W. A. Woyczyński, Representation of additive functionals and invariant characteristic functionals of linear processes-with independent pieces, Bull. Acad. Polon. Sci. Ser. Sci. Math. Astronom. Phys. 19 (1971), 221-230.

Department of Mathematics, Carnegie-Mellon University, Pittsburgh, Pennsylvania 15213

Current address: Institute of Mathematics, Wroclaw University, Wroclaw, pl. Grunwaldzki 2/4, Poland 